\title{
Biological behavior of the intramucosal Helicobacter pylori- negative undifferentiated-type early gastric cancer: comparison with Helicobacter pylori-positive early gastric cancer
}

\author{
Yusuke Horiuchi · Junko Fujisaki • Noriko Yamamoto • Tomoki Shimizu • Yuji Miyamoto • \\ Hideomi Tomida - Chika Taniguchi $\cdot$ Kenjiro Morishige $\cdot$ Masami Omae $\cdot$ Akiyoshi Ishiyama • \\ Toshiyuki Yoshio $\cdot$ Toshiaki Hirasawa Yorimasa Yamamoto Tomohiro Tsuchida • \\ Masahiro Igarashi · Toshifusa Nakajima · Hiroshi Takahashi
}

Received: 11 September 2014/ Accepted: 24 November 2014/Published online: 10 December 2014

(C) The International Gastric Cancer Association and The Japanese Gastric Cancer Association 2014

\begin{abstract}
Background The differences in the growth morphology, proliferative ability, and background mucosa of the cancer between Helicobacter pylori (HP)-positive (HP+) gastric cancer (GC) and HP-negative (HP-) GC are still unclear. To clarify the differences, we compared the characteristics of the two types of cancer.

Methods Of the 91 patients with undifferentiated-type early GC who underwent endoscopic treatment at our hospital between August 2005 and April 2011, 23 HP- GC patients (all of whom had signet ring cell carcinoma measuring $20 \mathrm{~mm}$ or less in diameter) and $46 \mathrm{HP}+\mathrm{GC}$ patients with signet ring cell carcinoma measuring $20 \mathrm{~mm}$ or less in diameter (out of a total of $68 \mathrm{HP}+\mathrm{GC}$ patients) were enrolled in this study. Endoscopic atrophy and background mucosa were classified according to the updated Sydney system. The proliferative capacity of the
\end{abstract}

Y. Horiuchi $(\bowtie) \cdot$ J. Fujisaki · T. Shimizu · Y. Miyamoto ·

H. Tomida $\cdot$ C. Taniguchi $\cdot$ K. Morishige $\cdot$ M. Omae $\cdot$

A. Ishiyama - T. Yoshio $\cdot$ T. Hirasawa - Y. Yamamoto .

T. Tsuchida $\cdot$ M. Igarashi

Department of Gastroenterology, Cancer Institute Hospital,

3-10-6 Ariake, Koto-ku, Tokyo 135-8550, Japan

e-mail: yusuke.horiuchi@jfcr.or.jp

N. Yamamoto

Department of Pathology, Cancer Institute Hospital, Tokyo, Japan

T. Nakajima

Department of Gastrointestinal Surgery, Cancer Institute

Hospital, Tokyo, Japan

H. Takahashi

Department of Gastroenterology, Showa University Fujigaoka

Hospital, Yokohama, Japan cancer was assessed by examining the MIB-1 labeling index.

Results With regard to the growth in the mucosal layer, the proportion of patients with cancer confined to the proliferative zone was significantly higher in the HP- GC group. Moderate or severer atrophy, intestinal metaplasia, mononuclear cell infiltration, and neutrophil infiltration according to the updated Sydney system were significantly commoner in the HP+ GC patients. Also, the MIB-1 labeling index was significantly higher in the HP+ GC group.

Conclusion HP+ GC appeared to show a higher proliferative capacity, more extensive spread, and more rapid progression, and inflammation associated with HP infection was suggested to be involved in the proliferation of this type of GC.

Keywords Gastric cancer - Undifferentiated-type carcinoma $\cdot$ Helicobacter pylori $\cdot$ Endoscopic gastrointestinal surgery

\section{Introduction}

Most patients with gastric cancer (GC) have a positive test result for Helicobacter pylori (HP) infection [1, 2]. Uemura et al. [1] reported, on the basis of a 9-year prospective study, that there were no cases of cancer among HP-negative $(\mathrm{HP}-)$ patients. However, later studies have shown the existence of a small number of patients with HP- GC; according to one report, an estimated $0.66 \%$ of GC patients test negative for HP infection [3]. An investigation conducted at our hospital of patients who underwent endoscopic submucosal dissection (ESD) also revealed the existence of a small number of HP- GC patients. According to a previous study, many of the $\mathrm{HP}-\mathrm{GC}$ 
patients had undifferentiated-type cancer [3]. Differentiated-type cancers include gastric cardia cancer, which is difficult to differentiate from Barrett's esophageal adenocarcinoma originating from short-segment Barrett's esophagus and gastric adenocarcinoma of the fundic gland type. Gastric cardia cancer was excluded from this study because the possibility of Barrett's esophageal adenocarcinoma could not be completely ruled out, which could potentially lead to the accumulation of incorrect data on GC. No cases of gastric adenocarcinoma of the fundic gland type were found during the study period. Therefore, all patients in whom definite HP- GC was diagnosed at our hospital had undifferentiated-type cancer (all of them had signet ring cell carcinoma measuring $20 \mathrm{~mm}$ or less in diameter). So far, the differences in cancer growth morphology or background mucosa according to the presence/ absence of HP infection have not been investigated histopathologically, and the differences in the characteristics between HP-positive (HP+) GC and HP- GC are unclear. Therefore, in this study, we extracted patients with undifferentiated-type early GC with signet ring cell carcinoma measuring $20 \mathrm{~mm}$ or less in diameter from among the HPGC patients and histopathologically compared them with HP+ GC patients with signet ring cell carcinoma measuring $20 \mathrm{~mm}$ or less in diameter to clarify the differences in the growth morphology, proliferative capacity, and background mucosa between the two types of cancer.

\section{Patients and methods}

The purpose of this study was to clarify the differences in the growth morphology of undifferentiated-type early GC (signet ring cell carcinoma) in the mucosa, its proliferative ability, and gastritis in the background mucosa between $\mathrm{HP}+\mathrm{GC}$ and $\mathrm{HP}-\mathrm{GC}$.

Ninety-one patients with undifferentiated-type early GC underwent ESD at our institution between August 2005 and April 2011. Of these, $23 \mathrm{HP}-\mathrm{GC}$ patients (all of who had signet ring cell carcinoma measuring $20 \mathrm{~mm}$ or less in diameter and intramucosal cancer) and $46 \mathrm{HP}+\mathrm{GC}$ patients with signet ring cell carcinoma measuring $20 \mathrm{~mm}$ or less in diameter, who had no history of eradication therapy and intramucosal cancer (out of a total of 68 patients with undifferentiated $\mathrm{HP}+\mathrm{GC}$ ) were enrolled in this study. HP- status was defined as the fulfillment of all of the following six criteria: no history of eradication therapy, negative result of the urea breath test, negative test result for HP antibodies, negative result of the pepsinogen test, endoscopically apparent regular arrangement of collecting venules (RAC) in the lower gastric body as diagnosed by three specialists in gastrointestinal endoscopy, and histological negativity for HP and inflammatory cell infiltration. Yagi et al. [4] reported that in standard endoscopy, RAC is visible as numerous minute red points in the corpus of the noninfected stomach, which cannot be visualized in HP gastritis. As a determinant of a normal stomach without HP infection, the presence of RAC showed $93.8 \%$ sensitivity and $96.2 \%$ specificity [5]. Moreover, Miki [6] reported that a positive pepsinogen test result was significantly more likely in patients with HP+ gastritis than in those with HP- gastritis. Pepsinogen is a serum marker of atrophic gastritis. HP status was defined as positive if at least one of the aforementioned six criteria was fulfilled. This study was approved by the Institutional Review Board of the Cancer Institute Hospital (Institutional Review Board no. 2013-1079).

Growth patterns in the mucosal layers were classified by periodic acid-Schiff staining of the ESD specimens into four categories: cancer limited to the proliferative zone, cancer in the mucosal surface layer to the proliferative zone, cancer present in the proliferative zone to the lower layer of mucosa, and cancer growing in all layers (Fig. 1) [7-9]. Atrophy was graded endoscopically according to the Kimura-Takemoto classification [10]. For the assessment of the background mucosa, histological grading of the ESD specimens was performed according to the updated Sydney system (USS) [11].

In addition, for investigation of the proliferative capacity, immunostaining was performed by the surface-activated bonding method using a mouse monoclonal antibody against human Ki-67 antigen (MIB-1, DAKO), which is a protein expressed in the cells of the proliferative cycle. The proportion of cells positive for MIB-1 antibody in the tumor cells was measured in two or more regions, with most frequent labeling of the nuclei, and the mean value was calculated to obtain the MIB-1 labeling index [12, 13], which was used to assess the cell proliferative capacity (Fig. 2).

Statistical analysis

Fisher's exact probability test was used to compare the two groups. The means and standard deviations of the tumor diameter and MIB-1 labeling index were calculated, and differences between the two groups were tested by the $t$ test and $F$ test, and when equal variances were not assumed, Mann-Whitney's $U$ test was applied. The statistical significance level was set at $p<0.05$. In addition, StatView, version 5.0 (SAS Institute, Cary, NC, USA), was used for the analysis.

\section{Results}

In relation to the patient characteristics, there were no significant differences in the sex ratio or age between the 


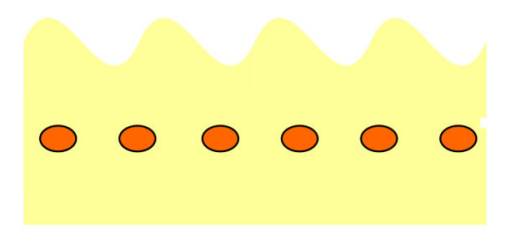

a Proliferative zone

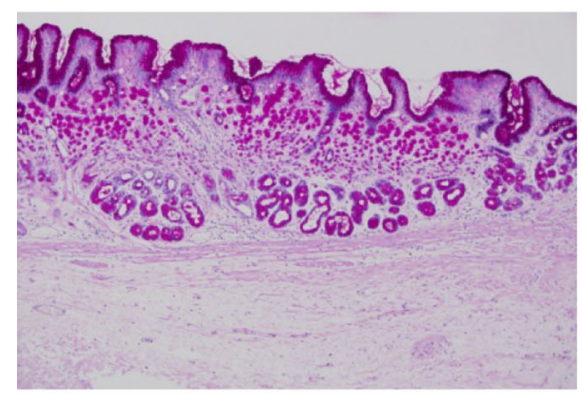

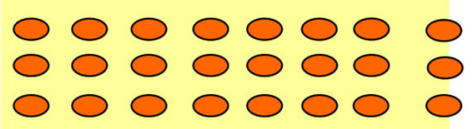

d All layers

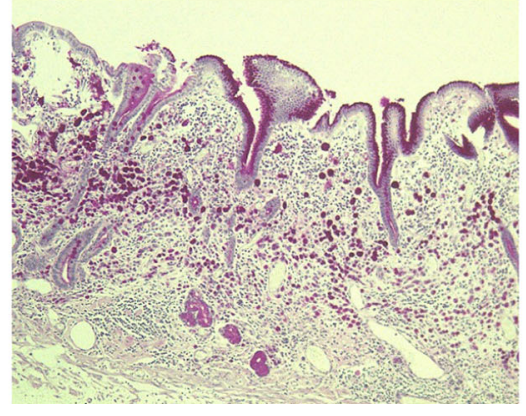

Fig. 1 Pathological growth patterns in undifferentiated-type cancer (intramucosal cancer): a cancer limited to the proliferative zone, b cancer present in the proliferative zone to the surface layer, $\mathbf{c}$ cancer

\section{$\because: 8: 8$}

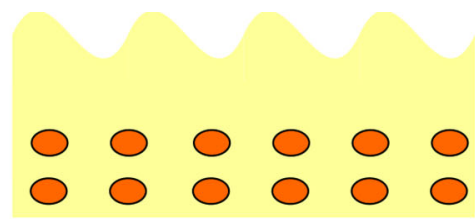

b Surface layer

to proliferative zone

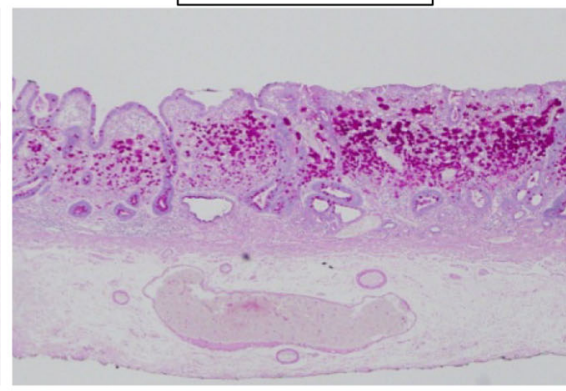

C Proliferative zone

to lower layer of mucosa

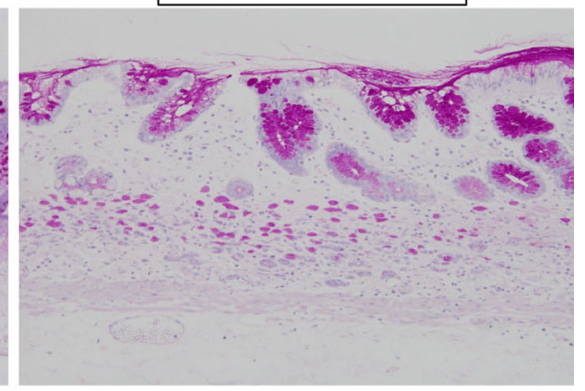

present in the proliferative zone to the lower layer of mucosa, and d cancer present in all layers
Fig. 2 MIB-1 labeling index. a Hematoxylin and eosin staining $(\times 40)$. b Ki-67 staining (MIB-1; $\times 40)$. MIB-1 labeling index was measured by the proportion of cells positive for MIB-1 antibody in the tumor cells in regions where the nuclei were labeled most frequently
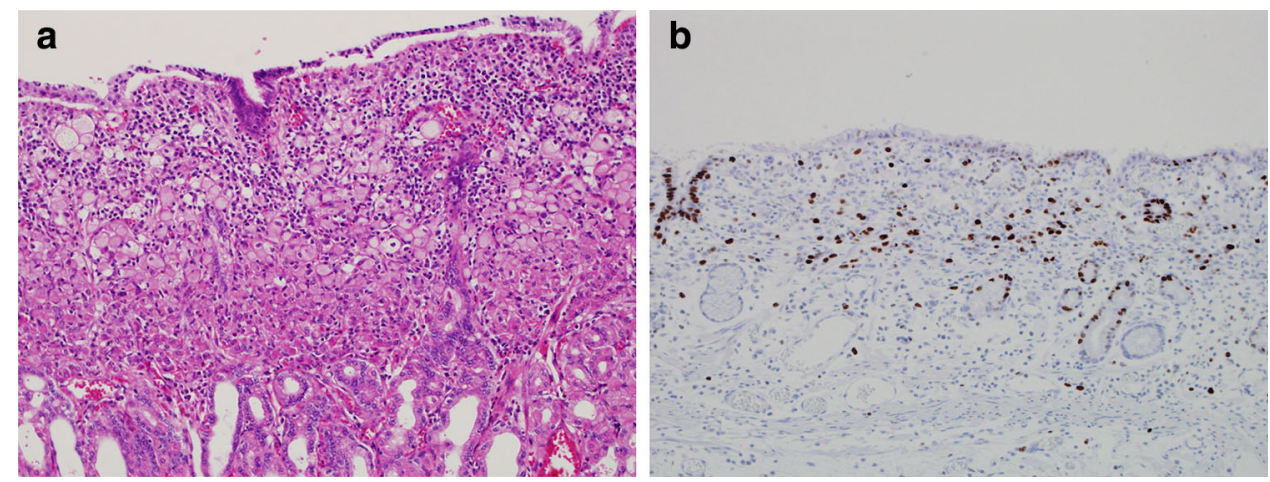

two groups. The mean tumor diameter was significantly smaller in the HP- GC group $(5.4 \pm 3.3 \mathrm{~mm})$ than in the $\mathrm{HP}+\mathrm{GC}$ group $(10.9 \pm 5.0 \mathrm{~mm})(p<0.05)$. With regard to the growth in the mucosal layer, the proportion of patients with cancer confined to the proliferative zone was significantly higher in the HP- GC group $(n=19$, $82.6 \%)$ than in the HP+ GC group $(n=14,30.4 \%)$ $(p<0.05)$. In contrast, the proportion of patients with 
cancer growing in all the layers was significantly lower in the HP- GC group $(n=1,4.3 \%)$ than in the HP+ GC group $(n=14,30.4 \%)(p<0.05)$ (Table 1).

Endoscopic atrophy was classified according to the Kimura-Takemoto classification as the open and closed types in $22 \mathrm{HP}+\mathrm{GC}$ patients $(48.8 \%)$ and $24 \mathrm{HP}+\mathrm{GC}$ patients $(52.2 \%)$, respectively, whereas it was classified as the closed type in all $23 \mathrm{HP}-\mathrm{GC}$ patients. Thus, the closed type was found at a significantly higher frequency in the HP- GC patients $(p<0.0001)$ (Table 2).

Histological grading was performed according to the USS to compare the background mucosa. The results revealed that moderate or severer atrophy, intestinal metaplasia, mononuclear cell infiltration, and neutrophil infiltration were significantly more frequent in $\mathrm{HP}+\mathrm{GC}$ than in HP- GC (Table 3).

In addition, the MIB-1 labeling index was determined by immunohistological assessment to determine the proliferative capacity of the cancer. The MIB-1 labeling index was significantly higher in the HP+ GC group (22.94 \pm $19.1 \%)$ than in the HP- GC group $(3.48 \pm 6.65 \%)$ $(p<0.0001)$ (Fig. 3).

\section{Discussion}

In this study, we extracted only HP- GC patients with signet ring cell carcinoma measuring $20 \mathrm{~mm}$ or less in diameter from among those with undifferentiated-type early GC who underwent ESD at our hospital, and selected HP+ GC patients with signet ring cell carcinoma measuring $20 \mathrm{~mm}$ or less in diameter as the control group. Previous studies have investigated HP- GC, including differentiated-type cancer, but it is difficult to reasonably compare these studies with our study $[3,6]$.

In regard to the accuracy of determining $\mathrm{HP}-$ status, some studies have referred to the incidence using only histopathology [14] or only anti-HP antibodies and the pepsinogen method [15], one determined it using anti-HP antibodies and histopathology, endoscopic findings, the pepsinogen method, and the urea breath test or the rapid urease test [3], and yet another determined it on the basis of histopathology, the rapid urease test, anti-HP antibodies, and a history of eradication therapy [16]. In contrast, we used six criteria [urea breath test results (negative), endoscopic findings, histopathology, pepsinogen test results, presence of anti-HP antibodies, and history of eradication therapy] in our study to select HP- patients. In some patients, long after eradication, various test results become negative, endoscopy shows RAC recovery, and histopathological examination shows improvement of inflammation and regeneration of the fundic glands, which make it difficult to differentiate these patients from those who have
Table 1 Patient characteristics

\begin{tabular}{|c|c|c|c|}
\hline & $\begin{array}{l}\mathrm{HP}+\mathrm{GC} \\
(n=46)\end{array}$ & $\begin{array}{l}\mathrm{HP}-\mathrm{GC} \\
(n=23)\end{array}$ & $p$ \\
\hline Male/female & $20: 26$ & $12: 11$ & NS \\
\hline Age (years) & $58.7 \pm 12.7$ & $52.6 \pm 8.4$ & NS \\
\hline \multicolumn{4}{|l|}{ Tumor location } \\
\hline Upper & $4(8.7 \%)$ & 0 & NS \\
\hline Middle & $27(58.7 \%)$ & $12(52.2)$ & NS \\
\hline Lower & $15(32.6 \%)$ & $11(47.8)$ & NS \\
\hline \multicolumn{4}{|l|}{ Tumor depth } \\
\hline Mucosa & $46(100 \%)$ & $23(100)$ & \\
\hline Submucosa & 0 & 0 & \\
\hline Lymphovascular invasion & 0 & 0 & \\
\hline \multicolumn{4}{|l|}{ Pathological growth patterns } \\
\hline Proliferative zone & $14(30.4 \%)$ & $19(82.6 \%)$ & $<0.05$ \\
\hline $\begin{array}{l}\text { Surface layer to } \\
\text { proliferative zone }\end{array}$ & $15(32.6 \%)$ & $3(13.0 \%)$ & NS \\
\hline $\begin{array}{l}\text { Proliferative zone to } \\
\text { lower layer }\end{array}$ & $3(6.5 \%)$ & 0 & NS \\
\hline All layers & $14(30.4 \%)$ & $1(4.3 \%)$ & $<0.05$ \\
\hline $\begin{array}{l}\text { Maximum tumor } \\
\text { diameter }(\mathrm{mm})\end{array}$ & $10.9 \pm 5.0$ & $5.4 \pm 3.3$ & $<0.05$ \\
\hline
\end{tabular}

$\overline{H P+G C \text { Helicobacter pylori-positive gastric cancer; } H P-G C}$ Helicobacter pylori-negative gastric cancer; NS not significant

Table 2 Assessment of endoscopic gastritis according to the Kimura-Takemoto classification

\begin{tabular}{lll}
\hline & \multicolumn{2}{l}{ Endoscopic gastritis } \\
\cline { 2 - 3 } & Open type & Closed type \\
\hline HP+ GC $(n=46)$ & $22(47.8 \%)$ & $24(52.2 \%)$ \\
HP- GC $(n=23)$ & 0 & $23(100 \%)$ \\
\hline
\end{tabular}

$\overline{H P}+G C$ Helicobacter pylori-positive gastric cancer, $H P-G C$ Helicobacter pylori-negative gastric cancer

Table 3 Comparison of the background mucosa between Helicobacter pylori-positive gastric cancer $(H P+G C)$ and Helicobacter pylori-negative gastric cancer $(H P-G C)$

\begin{tabular}{llrl}
\hline & HP+ GC & HP- GC & $p$ \\
\hline Atrophy & $43.5 \%(20 / 46)$ & $0 \%(0 / 23)$ & $<0.0001$ \\
Intestinal metaplasia & $19.6 \%(9 / 46)$ & $0 \%(0 / 23)$ & $<0.05$ \\
Neutrophils & $30.4 \%(14 / 46)$ & $0 \%(0 / 23)$ & $<0.005$ \\
Mononuclear cells & $67.4 \%(31 / 46)$ & $4.3 \%(1 / 23)$ & $<0.0001$ \\
\hline
\end{tabular}

Updated Sydney system-moderate to marked

not been infected with HP at all. Therefore, limiting the analysis to patients without a history of eradication therapy is likely to increase the accuracy of determining the HPstatus. 
Fig. 3 The MIB-1 labeling index. The vertical axis shows the number of patients and the horizontal axis shows the MIB1 labeling index. The MIB-1 labeling index was significantly higher in the Helicobacter pylori-positive $(\mathrm{HP}+)$ patients $(p<0.0001) . H P-$

Helicobacter pylori negative

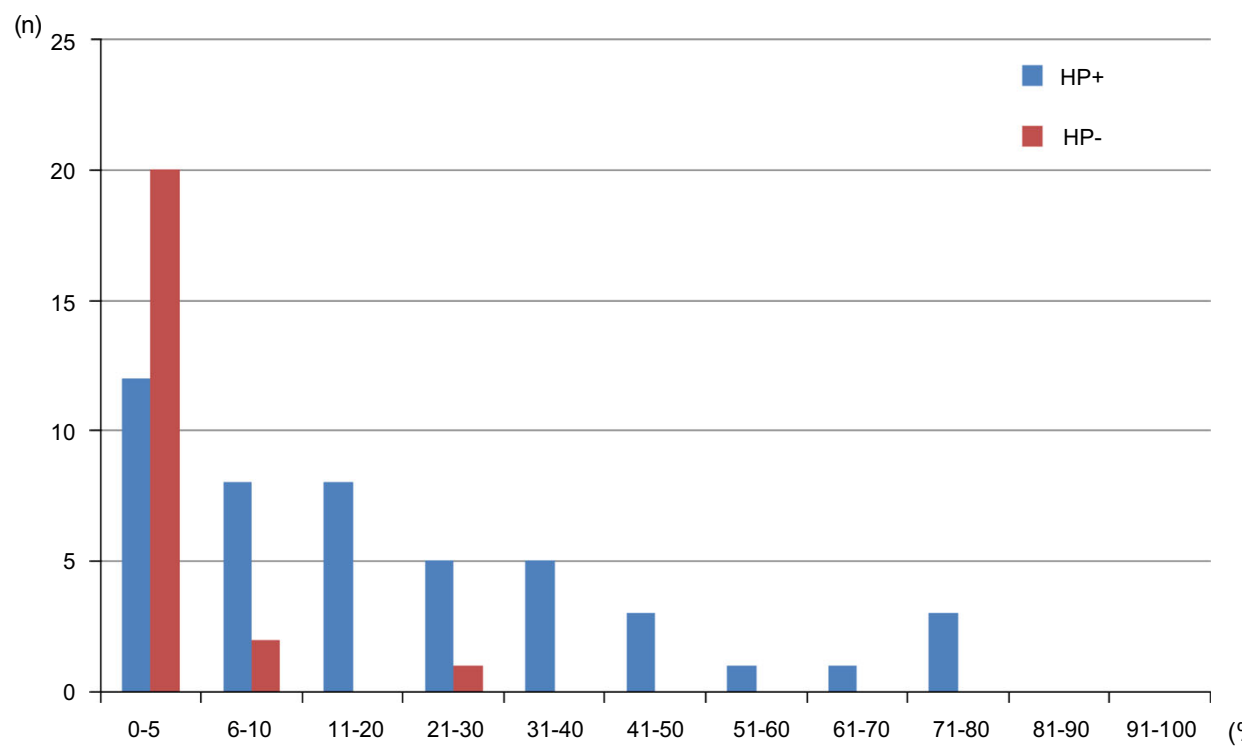

(\%)
In regard to the patient characteristics, there were no significant differences in the sex ratio or age between the $\mathrm{HP}+\mathrm{GC}$ and $\mathrm{HP}-\mathrm{GC}$ groups. According to our hospital data, undifferentiated-type early $\mathrm{GC}$ is common in young women, although the presence/absence of HP infection was not determined [17]. A study comparing patients with and without HP infection reported that $\mathrm{HP}$ - status was significantly commoner in female patients and that age was also significantly lower in HP- patients [3]. In the present study, the cancer size and histological type were limited to $20 \mathrm{~mm}$ or less in diameter and signet ring cell carcinoma, respectively, which was likely to yield results different from those described above. The mean tumor diameter was significantly smaller in the HP- GC group, and cancer confined to the proliferative zone was significantly commoner in the HP- GC group, suggesting the existence of differences in the intracellular proliferation and growth morphology between $\mathrm{HP}+\mathrm{GC}$ and $\mathrm{HP}-\mathrm{GC}$ patients, in that $\mathrm{HP}+\mathrm{GC}$ spreads more widely in the horizontal and vertical directions within the mucosa, thereby resulting in a larger tumor diameter. This was also supported by the significantly higher MIB-1 labeling index, which is an indicator of the intracellular proliferative capacity, in patients with $\mathrm{HP}+$ GC. That is, a high MIB-1 labeling index reflects a high proliferation capacity. It has been suggested that because of its higher proliferation capacity, HP+ GC spreads more widely in the horizontal and vertical directions within the mucosa, resulting in a larger tumor diameter. In contrast, because of the lower MIB-1 labeling index and lower proliferation capacity, $\mathrm{HP}-\mathrm{GC}$ is confined to the proliferative zone, resulting in a smaller tumor diameter. To date, no studies have reported the proliferative ability of $\mathrm{HP}-\mathrm{GC}$ or that of signet ring cell carcinoma. However, Fujiyama et al. [18] investigated the proliferative capacity of gastric mucosal epithelial cells using the bromodeoxyuridine labeling index in a Japanese macaque model, and reported a significant increase in the number of cells in the proliferative phase in HP-infected gastric mucosa as compared with that in non-HP-infected mucosa. In addition, it has also been reported that the MIB-1 labeling index was significantly lower in GC patients after eradication than in $\mathrm{HP}+$ GC patients [19, 20]. Although the profiles of the subjects in these studies were different from those in our study, the low proliferative capacity in non-HP-infected mucosa and the lower proliferative capacity in the $\mathrm{HP}$ - state generated by eradication seemed to be consistent with the results of our study.

In the comparison of the background mucosa, all the scores for atrophy, intestinal metaplasia, mononuclear cell infiltration, and neutrophil infiltration according to the Kimura-Takemoto and USS classifications were significantly higher in the HP+ GC group than in the HP- GC group, indicating severer inflammation of the background mucosa in $\mathrm{HP}+\mathrm{GC}$.

Taken together, the above findings and the findings of previous studies indicate that the proliferative capacity of the cancer is reactively enhanced owing to inflammatory cell infiltration in $\mathrm{HP}+\mathrm{GC}[1,19,21]$, which means that DNA synthesis and cell division occur actively in $\mathrm{HP}+$ GC. In contrast, inflammation is absent or very mild, and therefore, the proliferative capacity is also low in HP- GC. As a result, it has been suggested that $\mathrm{HP}+\mathrm{GC}$ spreads more widely in the horizontal and vertical directions within the mucosa, thereby causing a larger tumor diameter as compared with the tumor diameter caused by HP- GC.

A limitation of this study was that because the HP- GC patients enrolled in the study had signet ring cell carcinoma and did not include patients with differentiated-type 
cancer, such as gastric adenocarcinoma of the fundic gland type and gastric cardia cancer, the results of this study cannot be extrapolated to all HP- GC patients. Because all of the HP- GC patients enrolled in this study had signet ring cell carcinoma, $\mathrm{HP}+\mathrm{GC}$ patients with signet ring cell carcinoma were extracted for comparison. This was likely to generate a selection bias. However, limiting the investigated cases to signet ring cell carcinoma allowed elimination of the influence of the histological type, and thereby allowed more genuine results of comparison to be obtained between GC patients with and without HP infection.

\section{Conclusion}

The proliferative ability of cancer was higher in $\mathrm{HP}+\mathrm{GC}$ patients with signet ring cell carcinoma than in $\mathrm{HP}-\mathrm{GC}$ patients with signet ring cell carcinoma, and $\mathrm{HP}+\mathrm{GC}$ signet ring cell carcinoma does not remain in the proliferative zone, but spreads widely in the vertical and horizontal directions. Therefore, it can be speculated that $\mathrm{HP}+$ GC progresses more rapidly. This suggests that careful follow-up is necessary and HP eradication is important to prevent cancer spreading. In general, HP+ patients are followed up by endoscopy at 1- to 3-year intervals, whereas $\mathrm{HP}$ - patients without atrophic gastritis are followed up at 5-year intervals [17]. The results of this study are considered to provide a pathological basis in support of this practice.

Conflict of interest The authors declare that they have no conflict of interest.

\section{References}

1. Uemura N, Okamoto S, Yamamoto S, Matsumura N, Yamaguchi $\mathrm{S}$, Yamakido $\mathrm{M}$, et al. Helicobacter pylori infection and the development of gastric cancer. N Engl J Med. 2001;345:784-9.

2. Correa P. Helicobacter pylori and gastric carcinogenesis. Am J Surg Pathol. 1995;19:S37-43.

3. Matsuo T, Ito M, Takata S, Tanaka S, Yoshihara M, Chayama K. Low prevalence of Helicobacter pylori-negative gastric cancer among Japanese. Helicobacter 2001;16:415-419.

4. Yagi K, Honda H, Yang JM, Nakagawa S. Magnifying endoscopy in gastritis of the corpus. Endoscopy. 2005;37:660-6.

5. Yagi K, Nakamura A, Sekine A. Characteristic endoscopic and magnified endoscopic findings in the normal stomach without Helicobacter pylori infection. J Gastroenterol Hepatol. 2002;17:39-45.

6. Miki K. Gastric cancer screening by combined assay for serum anti-Helicobacter pylori $\operatorname{IgG}$ antibody and serum pepsinogen
levels-"ABC method". Proc Jpn Acad Ser B Phys Biol Sci. 2011;87:405-14.

7. Yao T, Fujiwara T, Watanabe E, Koga Y, Okada Y, Okabe N. Endoscopic diagnosis of expansion of gastric cancer. Stomach Intest. 1972;7:725-38 (in Japanese with English abstract).

8. Yao T, Mizoguchi M, Okada M, Imamura K, Nakabayashi S, Maeda K, et al. Endoscopic findings of early gastric cancer and comparison in resected specimens. Stomach Intest. 1988;23:55-66 (in Japanese with English abstract).

9. Okada K, Fujisaki J, Kasuga A, Omae M, Hirasawa T, Ishiyama A, et al. Diagnosis of undifferentiated type early gastric cancers by magnification endoscopy with narrow-band imaging. J Gastroenterol Hepatol. 2011;26:1262-9.

10. Kimura K, Takemoto T. An endoscopic recognition of atrophic border and significance in chronic gastritis. Endoscopy. 1969;3:87-97.

11. Dixon MF, Genta RM, Yardley JH, Correa P. Classification and grading of gastritis. The updated Sydney system. International Workshop on the Histopathology of Gastritis, Houston 1994. Am J Surg Pathol. 1996;20:1161-81.

12. Cattoretti G, Becker MGH, Key G, Duchrow M, Schlüter C, Galle J, et al. Monoclonal antibodies against recombinant part of the Ki-67 antigen (MIB-1 and MIB-3) detect proliferating cells in microwave-processed formalin-fixed paraffin sections. J Pathol. 1992;168:357-63.

13. Key G, Becker MGH, Duchrow M, Schluter C, Gerdes J. New Ki-67 equivalent murine monoclonal antibodies (Mib-1-3) prepared against recombinant parts of the Ki-67 antigen. Ann Cell Pathol. 1992;4:181-3.

14. Kakinoki R, Kushima R, Matsubara A, Saito T, Okabe H, Fujiyama $\mathrm{Y}$, et al. Mucin phenotype and background mucosa of intramucosal differentiated-type adenocarcinoma of the stomach. Oncology. 2004;66:379-87.

15. Kato S, Matsukura N, Tsukada K, Matsuda N, Mizoshita T, Tsukamoto T, et al. Helicobacter pylori infection-negative gastric cancer in Japanese hospital patients: incidence and pathological characteristics. Cancer Sci. 2007;98:790-4.

16. Yoon H, Kim N, Lee HS, Shin CM, Park YS, Lee DH, et al. Helicobacter pylori-negative gastric cancer in South Korea: incidence and clinicopathologic characteristics. Helicobacter. 2011;16:382-8.

17. Nakajima T, Yamaguchi T, Sano T. Database of gastric cancer, from Cancer Institute Hospital, Ariake. 2nd ed. Tokyo: Kanehara Shuppan; 2012. (CD-ROM file A).

18. Fujiyama K, Fujioka T, Murakami K, Nasu M. Effects of Helicobacter pylori infection on gastric mucosal defense factors in Japanese monkeys. J Gastroenterol. 1995;30:441-6.

19. Ito M, Tanaka S, Takata S, Oka S, Imagawa S, Ueda H, et al. Morphological changes in human gastric tumors after eradication therapy of Helicobacter pylori in a short-term follow-up. Aliment Pharmacol Ther. 2005;21:559-66.

20. Yamamoto K, Kato M, Takahashi S, Haneda M, Shinada K, Nishida U, et al. Clinico-pathological analysis of early stage gastric cancers detected after successful eradication of Helicobacter pylori. Helicobacter. 2011;16:210-6.

21. Tanaka A, Kamada T, Inoue K, Shiotani A, Kusunoki H, Manabe $\mathrm{N}$, et al. Histological evaluation of patients with gastritis at the high risk of developing gastric cancer using a conventional index. Pathol Res Pract. 2011;207:354-8. 